\title{
Vom Denkmalschutz zum Heimatschutz?
}

Die Rolle der FPÖ im städtebaulichen Diskurs anhand von zwei Wiener Beispielen

Im Zuge einiger Neu- und Umbauprojekte kam es in Wien in den letzten Jahren immer wieder zu heftigen Debatten um die Bewahrung alter Bausubstanz. In einigen Fällen haben sich Bürgerinitiativen gegründet, um geplanten Veränderungen etwas entgegenzusetzen. Nicht selten lässt sich beobachten, dass auch die Freiheitliche Partei Österreichs (FPÖ) versucht, sich aktiv in den Diskurs einzubringen und sich für den Erhalt bestimmter Gebäude einsetzt. Die FPÖ gibt sich volksnah und für den Denkmalschutz. Der folgende Text untersucht Online-Auftritte der FPÖ und analysiert die verschiedenen Verschränkungen von rechten Deutungsmustern im Denkmalschutzdiskurs. Anhand der Beispiele des „Café und Schloss Cobenzl“ und der „Steinhofgründe“ am Rande Wiens wird gezeigt, welche Strategien die FPÖ verfolgt, mit welchen Argumentationslinien sie arbeitet und welche Narrative produziert werden. Dabei zeigt sich, dass die Rechte jene Kontroversen besonders stark nutzt, die zugleich politisch und emotional aufgeladen sind. Vor allem in der Deutungshoheit von Geschichte in ihrer baulichen Manifestation wittern die Rechten Chancen.

\section{Einleitung}

Dass sich die FPÖ in Auseinandersetzungen um den Umgang mit historischer Bausubstanz in Wien einbringt, wirft für Denkmalschützer_innen, die sich als antifaschistisch verstehen, viele Fragen auf. Wieso ist dieses Thema für eine rechte Partei relevant und attraktiv? Welchen Erfolg verspricht sich die FPÖ davon? Dient der Kampf um alte Bausubstanz stellvertretend als Kampf um Tradition, Identität und kulturelle Hegemonie? Oder handelt es sich um trivialen Stimmenfang? Im vorliegenden Text wird untersucht, mit welchen Mitteln die FPÖ sich in Denkmalschutzdiskurse einbringt, und welche Narrative sie dabei anwendet. Mit einer kritischen Diskuranalyse (Jäger 1997) werden Stellungnahmen der FPÖ in Bezug auf zwei Wiener Beispiele untersucht: Das Schloss und Café Cobenzl, und die Steinhofgründe. Als Quellengrundlage dienen vor allem Wiener Presseaussendungen und Web-Veröffentlichungen. In diesen Verlautbarungen geht es darum, die eigene Zielgruppe zu erreichen, 
also (potentielle) Wähler_innen und Parteimitglieder. Der Zeitraum beschränkt sich auf die Jahre 2001 bis 2018. Im Zentrum stehen Narrative der FPÖ in Denkmalschutzbelangen und die Frage nach Querverbindungen zur übrigen politischen Ideologie der Freiheitlichen.

Zunächst werden potenzielle parteipolitische Interessen, die politische Lage auf Stadtebene sowie klassische rechte Taktiken beschrieben. Daraus werden fünf Vermutungen zu Zielen und Absichten der Freiheitlichen abgeleitet, die sie durch ihr Auftreten als Schutzmacht des Architekturerbes verfolgen. Im dritten Kapitel werden die Beispiele näher beschrieben und die darauf bezugnehmenden Beiträge der FPÖ untersucht. Die pikanten Verzweigungen der genannten Bauprojekte zu rechtsextremen Kreisen und der nationalsozialistischen Vergangenheit Wiens spielen dafür eine wichtige Rolle. Im letzten Teil werden die Textanalysen mit den im zweiten Kapitel formulierten Themenbereichen verbunden. Im Ergebnis wird der rechte Umgang mit Geschichte am Beispiel gebauter Strukturen greifbar.

\section{Die Wiener FPÖ und die Suche nach Polarisierung}

Die Themen Umbau, Neubau oder Grünflächenbebauung in urbanen Ballungsräumen provozieren Konflikte, die mitunter stark emotional ausgetragen werden. Das führt zur ersten Vermutung über parteipolitische Interessen an städtebaulichen Diskursen: Auch wenn nur eine kleine, unmittelbar negativ betroffene Gruppe (etwa die Nachbarschaft des geplanten Baugrundes) erreicht wird, können Mitglieder und Wählerstimmen gewonnen werden.

Die Situation der Wiener Stadtpolitik der letzten Jahrzehnte verleitet zur zweiten Vermutung: Trotz der insgesamt steigenden Popularität der Freiheitlichen in den letzten Jahren und der bundesweiten FPÖ-Regierungsbeteiligung seit 2017, gilt Wien immer noch als Hochburg der Sozialdemokratie und als weltoffene Metropole. Bei den Wiener Gemeinderatswahlen 2015 konnte die FPÖ bedeutende Erfolge verbuchen (Magistratsabteilung 62 2015). Dennoch ist die Dämonisierung der seit 2010 bestehenden rot-grünen Koalition für die FPÖ nicht einfach, wird doch stetig mit besten Ergebnissen bei Lebensqualität, Kriminalitätsrate und Mieten gepunktet (Magistratsdirektion 2018). Die Wiener Stadtregierung ist es allerdings auch, die Bauvorhaben begleitet oder Bauträger beauftragt, und damit maßgeblich das Stadtbild prägt. Wer mit diesbezüglichen Entscheidungen unzufrieden ist, kann den eigenen Unmut an die Wiener Roten und Grünen adressieren. Denkmalschutz- und Städtebaudiskurse können deshalb als ideale Projektionsfläche für Oppositionspolitik und Stimmungsmache gegen die aktuelle Stadtregierung Wiens dienen. Das Thema bietet also Angriffsfläche, um das rosige Bild von Wien zu hinterfragen.

Wie das Engagement der FPÖ in diesen Dingen zeigt, ermöglichen Bürgerinitiativen den direkten Kontakt zu Wähler_innen und - noch wichtiger - Präsenz auf der Straße. Bisher sind massenhafte Proteste aufgrund von Bauvorhaben zwar ausgeblieben, dennoch beinhaltet jeder Fall die Möglichkeit, bürgernahe Politik zu demonstrieren. Daraus folgt die dritte Vermutung: Auch vermeintlich aussichtslose und personell überschaubare Proteste dienen als Methode, um die Stimmung in der Wiener Bevölkerung weiter in Richtung Parteilinie zu rücken. 
Ein weiterer Vorteil der Themen Denkmalschutz und Städtebau ist die Verschränkung mit anderen Politikfeldern. Im Rahmen von Diskursen über Denkmalschutz werden auch Fragen zu Identität, (nationaler) Tradition, Kultur, Umweltschutz et cetera behandelt. Kulturpolitik als nationale Identitätsstiftung kann erhebliche Ausmaße annehmen und dabei helfen, die Geschichtsschreibung im eigenen Interesse zu transformieren. So können Kontinuitäten ohne Rücksicht auf historisch heikle Abschnitte der Vergangenheit geschaffen werden. Die vierte Vermutung betrifft also, neben der Verschränkung mehrerer Themen im Denkmalschutzdiskurs, vor allem das Potenzial historischer Deutungshoheit.

Die letzte Vermutung bezieht sich auf strategische Vorteile rechter Parteien gegenüber ihren ideologischen Gegner_innen.Aufmerksame Beobachter_innen können diskursive Strategien der Rechten entlarven und zerlegen. Bei vielen Themen sind die Absichten bekannt und die Positionen klar, etwa bei Migrationsfragen oder Gender-Debatten. Auch wenn der Schutz historischer Bausubstanz und damit die Rückbesinnung auf das Alte für rechte Akteur_innen naheliegt, ist Denkmalschutz in der Wahrnehmung großer Bevölkerungsteile kein klassisch rechtes Betätigungsfeld. Oberflächlich betrachtet erscheint das Feld Denkmalschutz als unbestellt und bietet Kritiker_innen weniger Angriffsfläche als andere rechte Positionen.

\section{Von Kulturschande und Baummorden}

\subsection{Der Fall Steinhof}

Das Gelände des Otto-Wagner-Spitals und der angrenzenden Steinhofgründe (kurz Steinhof) im heutigen 14. Wiener Gemeindebezirk wurde 1907 als Landes-Heil- und Pflegeanstalt eröffnet und gilt inklusive Sakralbau als Juwel des Wiener Jugendstils (Magistratsabteilung 8 und 9 2018a). Durch die Nutzung des Areals während des NS-Regimes erlangte es traurige Bekanntheit unter der Bezeichnung ,Am Spiegelgrund‘. Es war Schauplatz grausamer Experimente der nationalsozialistischen Tötungsmedizin an Menschen mit Behinderung oder psychischen Krankheiten (Gedenkstätte Steinhof 2018).

Die weitläufigen Grünanlagen der Steinhofgründe sollten bereits Ende der 1970er Jahre teilweise bebaut werden. 1981 wurde eine Volksbefragung durchgeführt, bei der sich rund 140.00o Menschen (etwa 53 Prozent der abgegeben Unterschriften) gegen die geplante Bebauung aussprachen - eine beachtliche Menge bei nicht einmal zwei Millionen Einwohner_innen. 1983 wurden weite Teile der Grünzone zum Schutzgebiet erklärt (Magistratsabteilung 49 2017). Mitte der 200oer Jahre gelangten erste Mitteilungen an die Öffentlichkeit, die von der Verlagerung einiger Krankenanstaltsabteilungen an andere Standorte in Wien berichteten, sodass einige Gebäude des Otto-WagnerSpitals leer stehen würden beziehungsweise für Wohnungen, Lokale oder Hotels geöffnet werden könnten. 2011 wurden jene Vorhaben konkreter. Bis 2019 sollten etwa 160 Wohnungen entstehen, womit Baumfällungen und signifikante Veränderungen des architektonischen Ensembles einhergehen würden beziehungsweise bereits geschehen sind (Krutzler 2017a). Dies provozierte teils heftigen Protest von Bürgerinitiativen (Initiative Kultur- und Naturerbe Wien 2015, Kadi 2016). 
Die FPÖ schaltete sich bereits 2011 in die Debatte ein und zeigte vor allem im Jahr darauf immer mehr Präsenz mittels Presseaussendungen. 2012 wurde ein Mediationsverfahren zwischen Stadt, Bürgerinitiative und Käufer_innen eingeleitet (Kadi 2016). In der dazu passenden Aussendung mit dem vielsagenden Titel „Erpresser-Methoden bei Steinhof-Mediation“ wird die schroffe Empörung der FPÖ deutlich:

„Es ist hoch an der Zeit, dass Bürgermeister Häupl der wie beim Parkpickerl auch hier auf Pjönjang-Kurs segelnden Planungsstadträtin das Steuerruder aus der Hand nimmt und die Richtung für die $\mathrm{Zu}-$ kunft des einzigartigen Jugendstil-Ensembles vorgibt. Die FPÖ wird daher in der nächsten Gemeinderatssitzung einen Antrag einbringen, dass Steinhof zur Chefsache wird und die Anstrengungen von Bürgerinitiativen und FPÖ zur Erhebung des Architektur-Juwels zum UNESCO-Welterbe auch offiziell von der Stadt unterstützt werden, kündigt Mahdalik an.“[1] (APA-OTS FPÖ-Wien 30.7.2012)

Abgesehen von der auffälligen und in der Wiener Politik bekannten Sprache des FPÖ-Sekretärs Toni Mahdalik, zeigen sich einige beliebte Muster der FPÖ-Rhetorik. Mit dem sogenannten Parkpickerl (kostenpflichtige Plakette, um ein Auto in bestimmten Bezirken zu jeder Tages- und Nachtzeit abstellen zu dürfen) wird auf eine weitere Kontroverse in der Wiener Stadtpolitik angespielt, die viele Gemüter erhitzt hat. Mit dem ,Pjöngjang-Kurs‘ unterstellt die FPÖ der Planungsstadträtin offenkundig autoritäre Politikpraktiken. Umgekehrt würde es eher zu einem Top-down-Politikmodell passen, die Steinhof-Causa zur Chefsache zu machen.

Im Frühjahr 2017 meldete sich die FPÖ mehrmals über ihre Webpage und Presseaussendungen zu Wort, als die ersten Bäume gefällt wurden. Der Website-Eintrag mit dem Titel „Freiheitliche Mandatare protestieren gegen Umweltzerstörung und Profitgier“" zeugt von pathetischen Narrativen und fürsorglicher Stellvertreterpolitik:

„RRRRRRRRRRRRRR. Motorsägenlärm zerreist die Stille. Über Steinhof hallt das Kreischen der Kettensägen, Metall gegen hunderjähriges Holz. Baummord für Profit. In den frühen Morgenstunden des Valentinstags 2017 werden Fakten geschaffen. Bäume fallen, der Boden bebt und die Penzinger Bürger müssen hinter Gittern der Vernichtung eines Teils ihres Naherholungsgebiets beiwohnen. Heimlich wollte man die Schlägerungen durchführen doch Vertreter der Bürgerinitiative und Bezirksträte der FPÖ Penzing, FPÖ Ottakring sowie FPÖ Wien Stadtrat Toni Mahdalik sind trotz der Nacht und Nebel Aktion vor Ort und dokumentieren die Umweltzerstörung. So zeigt sich wieder: Umweltschutz ist Heimatschutz und vor allem die FPÖ kämpft mit den Bürgern gegen diesen miesen Anschlag auf unsere Lebensqualität und den Raub an der Lebensqualität zukünftiger Generationen." (FPÖ Wien-Penzing 2017)

Sphärischer Einstieg. Fast liest sich der Text wie eine Mischung aus Thriller und Trivialroman. Auffällig und zugleich klassisch ist die Bezeichnung ,Mord‘ für das Fällen von Bäumen, garniert mit Vernichtung, Zerstörung, Anschlag, Raub. Die FPÖ-Mandatare werden als Helden gefeiert, die sich auf 
Augenhöhe mit den Bürgern für die viel zitierte Lebensqualität in Wien stark machen. Die Gegenüberstellung von Natur und Profit gibt dem freiheitlichen Narrativ einen antikapitalistischen Anstrich. Auch der Heimatbegriff findet seinen Platz in der Parole „Umweltschutz ist Heimatschutz“. Ein traditionell grünes Thema wird mit Heimat verknüpft.

Am Tag der Veröffentlichung dieses Textes wurde eine Presseaussendung verschickt, in der die FPÖ mit einer Strafanzeige droht (APA-OTS FPÖWien 14.2.2017). Die Baumfällungen vom 14. Februar 2017 werden als „rot-grünes Valentins-Massaker am Steinhof" bezeichnet. Wieder ein Wort, das gewöhnlich für grausamen Massenmord an Menschen steht. Außerdem betont der Text erneut die Anwesenheit der FPÖ-Politiker neben Vertreter_innen einer Bürgerinitiative während der frühen Morgenstunden auf den Steinhofgründen.

Im April 2017 folgen weitere Aussendungen zum Baubeginn im östlichen Teil des Areals (APA-OTS FPÖ-Wien 11.4.2017 und 19.4.2017). Darin wird näher auf das architektonische und damit historische Erbe eingegangen:

„Heute vor 99 Jahren ist der,Weltstadtarchitekt‘ Otto Wagner gestorben, dem das Wien Museum 2018 eine große Ausstellung widmen wird. Gestern haben SPÖ und Grüne mit der Zerstörung des weltweit einzigartigen Jugendstil-Ensembles begonnen, damit ein stadteigener Wohnbauträger exklusive Wohnungen in grüner Bestlage aus dem Boden stampfen kann!“ (APA-OTS FPÖ-Wien 11.4.2017)

Die Selbstdarstellung der FPÖ als Partei der kulturellen Bewahrung, die als einzige das Verständnis für architektonische Besonderheiten aufbringt, wird unterstrichen und zu aktuellen Kulturangeboten in Verbindung gebracht. Zudem wird die Bebauung als „Kulturschande“ bezeichnet und ein Baustopp gefordert, „um das Erbe Otto Wagners unversehrt für die Nachwelt zu erhalten“. Einen der namhaftesten österreichischen Architekten als Opfer der Stadtregierung hinzustellen, zeigt die taktisch kluge Verschränkung verschiedener Ebenen.[2]

Die FPÖ bedient im Falle Steinhof vor allem die Rolle als Oppositionspartei der Wiener Regierung. Tatsächlich sind bei diesem Bauprojekt viele Fragen offen, viele Vorgänge schlecht nachvollziehbar und die Argumentation von Teilen der Stadtregierung kaum befriedigend. Insofern bietet das Beispiel der Steinhof-Bebauung genügend Angriffsfläche, um die rot-grüne Koalition in Bedrängnis zu bringen. Die Präsenz der FPÖ-Politiker am Schauplatz und die Unterstützung einer der beiden involvierten Bürgerinitiativen verstärken den Eindruck einer ernst gemeinten bürgernahen Politik. Immer wieder wird auf die international anerkannte Bedeutung der Jugendstil-Anlage verwiesen und dass diese zum UNESCO-Weltkulturerbe erhoben werden müsse (APA-OTS FPÖ-Wien 3.4.2013). Zugleich fordert FPÖ-Bundesparteiobmann Heinz-Christian Strache, die Nutzung des Areals für soziale, medizinische und kulturelleZwecke zu öffnen - Forderungen, die in Anbetracht der aktuellen türkis-blauen Bundesregierung nahezu zynisch erscheinen, da genau jene Bereiche massiven Kürzungen ausgesetzt sind. Doch auch die Verschränkung mit Unterthemen der FPÖ-Agenda gelingt an vielen Stellen. Das an sich geringe Umweltbewusstsein der Partei rückt in den Vordergrund und wird mit dem Heimatbegriff in Verbindung gebracht. 
Doch worüber spricht die FPÖ im Zusammenhang von Steinhof nicht? Wenig überraschend wird die NS-Vergangenheit konsequent ausgespart. Ein Schlaglicht auf die Auseinandersetzung der FPÖ mit der Geschichte der Steinhofgründe zur NS-Zeit wirft die Diskussion rund um Friedrich Zawrel, einen Überlebenden der Gräueltaten am Spiegelgrund. Als ihm zu Ehren eine Schule umbenannt werden sollte, reagierte die FPÖ empört und bezeichnete Zawrel als Kriminellen, der kein Vorbild für Schüler_innen sein könne (Klenk 2015). Eine reflektierte und respektvolle Aufarbeitung des Tatorts Spiegelgrund wäre im Falle einer FPÖ-Entscheidungsmacht nicht zu erwarten. Andererseits wird der sonst inflationäre Rekurs auf Migrationsthemen von der FPÖ in diesem Fall kaum praktiziert. Sie fokussiert auf die Themen Umwelt und kulturelles Erbe. Der Brückenschlag zur sonst stets präsenten, ablehnenden Haltung gegenüber Nicht-Österreicher_innen wird womöglich der mit der Partei kooperierenden Bürgerinitiative „Steinhof erhalten“ überlassen. Diese verlautbart: „Einige Pavillons wurden zur Unterbringung von Asylanten genutzt. Wir wünschen uns aber eine andere soziale Nutzung, für Alte und Kranke“ (Schuh 2016).

\subsection{Der Fall Cobenzl}

Der Cobenzl ist einer der Hausberge Wiens. Der Name wird jedoch oft mit dem Grundstück des Schlosses und Cafés am Cobenzl gleichgesetzt. Die Geschichte dieses Areals geht bis ins 16. Jahrhundert zurück. Bedeutend für die heutige Nutzung ist vor allem die Phase des Besitzers Graf Johann Philipp Cobenzl im späten 18. Jahrhundert. Er ließ die zuvor errichteten Häuser des Jesuitenordens zu einem großen Schloss umbauen, ergänzte es durch eine Meierei und eine Gartenanlage und machte den Ort für die Öffentlichkeit zugänglich. Zu dieser Zeit hat sich auch die Bezeichnung „Am Cobenzl“ bei der Wiener Bevölkerung durchgesetzt. Das vom Grafen erbaute Schloss hat seitdem verschiedenen Zwecken gedient und wurde mehrfach umgebaut, bis es Anfang des 20. Jahrhunderts in den Besitz der Stadt Wien gelangte. Das Gebäude verfiel immer mehr und wurde schließlich 1966 großteils abgetragen. Der spätere Pächter Olaf Auer belebte das Gelände wieder und erbaute in den 1980ern das bis heute bestehende Schlösschen im barocken Stil (Magistrastabteilungen 8 und 9 2018b).

2012 meldete die Stadt Wien beziehungsweise das Forstamt Eigenbedarf an und übermittelte dem Pächter die Kündigung. Jahrelange juristische Auseinandersetzungen folgten (Laubner 2016). Am Ende setzte sich die Stadt mittels Zwangsräumung durch, und Auer musste den Cobenzl im März 2017 verlassen (Krutzler 2017b). Die Stadt plant, den Standort zu modernisieren und will sich an den bereits bestehenden Strukturen und den vergangenen Nutzungen orientieren.

Unverzüglich nach der Räumung Auers meldete sich die „Initiative Denkmalschutz" zu Wort und forderte die Erhaltung der Bausubstanz auf dem Schlossareal (APA-OTS Initiative Denkmalschutz 2017). Die historisch bedeutsamen Gebäudeteile stehen bis heute nicht unter Denkmalschutz und könnten dem geplanten Umbau zum Opfer fallen. Die Initiative Denkmalschutz hat zu diesem Zwecke eine Petition gestartet und die Denkmalschutzbehörden um Prüfung des Baubestandes gebeten. Auch die FPÖ hatte sich im 
Zeitraum der drohenden Räumung zum Thema geäußert und sogar zu einer Demonstration aufgerufen. Einen Monat vor Auers erzwungenem Auszug wurde auf der Website der FPÖ Wien über die Aktion berichtet:

„Sage und schreibe 34 Jahre steckte Olaf Auer als Pächter von Schloss Cobenzl sein gesamtes Vermögen und viel Herzblut in den Erhalt des Anwesens. Nun will ihn die Stadt Wien mit allen Mitteln loswerden. Das Schloss möglicherweise sogar abgerissen werden. Die Stadt sucht international bereits einen neuen Pächter. Die FPÖ-Wien versucht dies zu verhindern und kämpft an der Seite mehrerer Bürgerinitiativen für den Erhalt von Schloss Cobenzl!“ (FPÖ Wien 2017)

Auf dem dazugehörigen Foto sind Vertreter der FPÖ Wien und der Pächter Olaf Auer zu sehen. Fast alle sind mit Schildern der FPÖ und einem Transparent mit der Aufschrift „Retten wir Schloss Cobenzl“ ausgestattet. Wer bei dieser Gelegenheit die Bürgerinitiativen repräsentierte, ist nicht zu erkennen. Zudem stellt sich die Frage, welche Initiativen gemeint sind, da nur die Initiative Denkmalschutz erwähnt wird. Der Pächter ist das unterstützenswerte Opfer. Schließlich ist er es, der sich der Stadtregierung entgegenstellt.

Die Hügel am Cobenzl sind nicht nur unter Spaziergänger_innen und Ausflügler_innen beliebt. Auch deutschnationale Burschenschafter halten dort immer wieder Sonnwendfeiern ab. Das Dokumentationsarchiv des österreichischen Widerstandes (DÖW) hat über die Jahre 2002, 2004 und 2007 berichtet, in denen die rechtsextreme Österreichische Landsmannschaft (ÖLM) und der Wiener Korporationsring jeweils am 21. Juni zur Feier am Cobenzl einluden (DÖW 2002 bis 2016). Traditionell wird zu diesem Anlass eine ,Feuerrede' gehalten, welche die deutschnationale Gesinnung der Anwesenden widergibt. Das DÖW erwähnt im Beitrag des Jahres 2002 auch den Pächter des Cobenzl-Schlosses: „Auf dem Grundstück des als ,Kameraden' titulierten Olaf Auer trafen sich rund 150 Personen aus dem ,Dritten Lager', darunter vor allem Burschenschafter, FPÖ-Mandatare und auch ein paar jener Skinheads, welche zuletzt am 13. April am Heldenplatz und in der Kärntnerstraße öffentlich ihre Gesinnung zur Schau stellten.“ (DÖW 2002) Interessanterweise wurde der Standort der Sonnwendfeier im Jahr 2016 vom Cobenzl zur Windischhütte im Wienerwald verlegt. Auch hier spielt der Cobenzl-Pächter eine Rolle. Das DÖW fasst die Umstände der Übersiedlung folgendermaßen zusammen: „Den Hintergrund für den Verlust des günstiger gelegenen Veranstaltungsortes dürfte der Rechtsstreit bilden, in welchem sich der Pächter des gemeindeeigenen Schlosses und Cafés am Cobenzl, Olaf Auer, gerade mit der Stadt Wien befindet. Jedoch bietet die ÖLM gratis Transferbusse zur Windischhütte an, die Kosten dafür wurden von der Wiener FPÖ übernommen. " Man könnte meinen, dass die Präsenz von Burschenschaftern rund um Auers Gelände in dieser heißen Phase der Auseinandersetzung kein vorteilhaftes Bild abgegeben hätte.

Seit dem Abzug Auers ist das Gelände für rechtsextreme Kreise jedenfalls kein einfaches Pflaster mehr. Die neurechte Identitäre Bewegung hat bereits öfters versucht, Märsche am Kahlenberg und Cobenzl abzuhalten. Dabei kam es jedoch stets zu Protesten. Die Zeiten eines mit den Rechten kooperierenden Pächters am Cobenzl waren vorbei. 
Im Fall des Cobenzl hat sich die FPÖ nicht so häufig zu Wort gemeldet wie in der Debatte zu den Steinhofgründen. Jedoch sind die persönlichen Verbindungen umso stärker, und die Haltung gegenüber der Stadtregierung ist dieselbe. Offenbar erschien es wenig lohnend, mit Anwohner_innen oder Umbau-Gegner_innen zusammenzurücken. Die Beziehung der FPÖ zum Gebäude am Cobenzl drückt sich nicht primär auf der diskursiven Ebene aus. Der Fall zeigt viel eher, welche Ausmaße eine FPÖ-Hegemonie in Wien bei Vergabe, Verwaltung und Nutzung von Grundstücken und besonderen Bauten hätte.

\section{Die Vergangenheit für die Zukunft nutzen}

Die Beispiele zeigen nur einen Ausschnitt. Welche Zukunftsaussichten im Falle einer freiheitlichen Entscheidungsmacht in Wien lassen sich annehmen? Beinhaltet das Thema Denkmalschutz virulente Anknüpfungspunkte? Im Kontext der eingangs formulierten Vermutungen soll abschließend gezeigt werden, welche Anknüpfungspunkte Rechte in städtebaulichen Debatten finden und welche Stellungnahmen diesbezügliche Absichten erkennen lassen.

DieFPÖ Wien hat bei beiden Bauprojekten stets ihre Präsenz vor Ort und ihren gemeinsamen Protest mit Bürgerinitiativen hervorgehoben. Und das, obwohl die Initiativen kaum aktive Unterstützung der FPÖ zum Ausdruck brachten. Auf jeden Fall versuchen die Web-Veröffentlichungen und Aussendungen der FPÖ zu vermitteln, dass die Partei ihren Selbstzuschreibungen von Bürgernähe, direkter Demokratie und Interessenvertretung des ,kleinen Mannes gerecht wird. Die Emotionalität, die oft mit städtebaulichem Wandel einhergeht, passt zudem gut zur ohnehin aufgeladenen Rhetorik der FPÖ. Außerdem können die Freiheitlichen bei den Protesten gegen Bauvorhaben der Stadtregierung auf ein Alleinstellungsmerkmal hoffen. Weder die Grünen noch die Sozialdemokrat_innen (SPÖ) werden gegen ihre eigenen Beschlüsse auf die Straße gehen, und auch die Österreichische Volkspartei (ÖVP) als in Wien tendenziell schwache Kraft widmet sich dem Thema kaum. Die FPÖ kann das Feld Denkmalschutz und Städtebau und den damit zusammenhängenden Protest also gut für sich nutzen, um als engagierte Bürgerpartei sichtbar zu werden. Durch die vordergründige Beschäftigung mit architektonischem Erbe und baulichen Veränderungen spricht die FPÖ außerdem viele Personen an, die hinsichtlich anderer Fragen (etwa der Migrations- oder Sozialpolitik) nicht von der Partei überzeugt sind.

Deutlich erkennbar ist, dass der Aktionismus im städtebaulichen Bereich die ideale Projektionsfläche für Oppositionspolitik darstellt. Die Betonung städtischer Misswirtschaft wirkt glaubhaft, da des Öfteren schwer nachvollziehbare Entscheidungen in der Wiener Baupolitik getroffen werden. Sprachlich wird dies durch eigenwillige Komposita à la ,rot-grünes ValentinsMassaker" transportiert. Die Verschränkung von Denkmalschutz mit der Rolle einer vehementen Oppositionspartei ist offenkundig - gepaart mit heftig demagogischer Sprache.

Vor allem im Fall Steinhof wird die Verbindung zum Umweltschutz stark gemacht. Das Thema veranschaulicht zugleich den freiheitlichen Opportunismus: Der Streit um ein Kraftwerk an der Mur in Graz hatte zur Auflösung der Stadtregierung von Volkspartei und Kommunistischer Partei und zu 
einer Protestwelle geführt. Anschließend kam es zur Bildung einer schwarzblauen Koalition, die das Bauprojekt vorantrieb und zur Umsetzung verhalf. Tausende Bäume fielen dem Großprojekt zum Opfer. Kritik an dieser Zerstörung wurde mit dem Verweis auf ausreichende Ersatzpflanzungen abgetan (Bonvalot 2018). Würde sich die FPÖ dem Thema Umweltschutz konsequent widmen, wäre eine Zustimmung zu diesem Projekt eigentlich unmöglich. Auch der Denkmalschutz an sich ist für die FPÖ kein unterstützenswertes Anliegen. In Bezug auf einen etwaigen Neubau des FußballNationalstadions in Wien äußerte sich Parteichef und aktueller Sportminister H. C. Strache abfällig über den im Wege stehenden Denkmalschutz: „Das Happel-Stadion gehört niedergerissen [...], dieser unsinnige Denkmalschutz darf nicht schlagend werden. "(Huber 2018) Dabei war es die FPÖ selbst, die während der Regierungsbeteiligung Anfang der 2000er Jahre das Stadion (1931 erbaut) unter Schutz stellte (ebd.).

Viel berechenbarer erscheinen die politischen Absichten im Umgang mit Denkmälern und kulturellem Erbe. Namhafte Vertreter_innen der österreichischen Kunst wie Otto Wagner gelten als ehrwürdig und dienen als Aushängeschild für nationale und künstlerische Errungenschaften. Darauf stützt die FPÖ ihre Argumentation für den Schutz der Steinhof-Anlage. Dass das Gelände aufgrund der Nutzung in der NS-Zeit belastet ist und eher Gedenk- und Bildungszwecken dienen sollte, würde die FPÖ wohl kaum als Forderung formulieren.

Abschließend ist der Umgang der FPÖ mit Denkmalschutz, mit historischem sowie architektonischem Erbe in einen größeren Zusammenhang zu stellen. Die FPÖ hat mit ihren diesbezüglichen Interventionen noch keine entscheidenden Erfolge verbuchen können, sei es auf der Ebene des öffentlichen Protests oder hinsichtlich der Abwendung baulicher Vorhaben. Trotzdem scheint es der Partei weiterhin ein Anliegen, Präsenz zu zeigen, Widerstand zu leisten und eigene Narrative durch Wortbeiträge und teils aufwendige Inszenierungen zu verbreiten. Auch die der FPÖ ideologisch nahestehende Partei Alternative für Deutschland (AfD) setzt sich regelmäßig für verstärkten Denkmalschutz und die Betonung des kulturellen Erbes ein. Und die Bedeutung all dessen würde in beträchtlichem Maße zunehmen, wenn rechte Parteien an die Macht gelangten. Die Emotionalisierung und Entsachlichung der Argumentation spielt eine wichtige Rolle. Diese klassischen Taktiken Rechtsextremer werden auch auf der Ebene erbauter Strukturen angewendet und avancieren zu einem Instrument der eigenen Geschichtsschreibung. Nicht nur Vergangenes würde der rechten Auslegung unterzogen. Auch das Bestehende könnte zusätzlich zur gewünschten historischen Kontinuität umgedeutet werden. Die Fälle Cobenzl und Steinhof geben einen Vorgeschmack darauf, welche Nutzungen Wiener Immobilien (und deren Umgebung) widerfahren könnte, wenn deutschnationale Burschenschafter darüber entscheiden, welche Bauten stehenbleiben, wie sie verändert werden, was sichtbar oder unsichtbar gemacht wird und wer sie nutzen kann. 


\section{Endnoten}

[1] Hinweis zu allen Zitaten der FPÖ: Rechtschreibfehler und fehlende Satzzeichen, wie etwa Beistriche und Anführungszeichen, wurden von den originalen Textquellen übernommen.

[2] Was hier nicht erwähnt wird, ist, dass die FPÖ im Jahre 2006 selbst noch für die Umwidmung der Flächen am Steinhof gestimmt hat (Wiener Zeitung 2011).

\section{Autor_innen}

Magdalena Augustin ist Kunsthistorikerin und arbeitet zu den Themen Stadtkultur, kritische Denkmalpflege, Jugend- und Gegenkultur.

magdalena.augustin@igkulturwien.net

\section{Literatur}

APA-OTS FPÖ-Wien (30.7.2012): FP-Mahdalik: Erpresser-Methoden bei SteinhofMediation? https://www.ots.at/presseaussendung/OTS_20120730_OTSo041/fpmahdalik-erpresser-methoden-bei-steinhof-mediation (letzter Zugriff am 5.12.2018).

APA-OTS FPÖ-Wien (3.4.2013): Strache kritisiert Expertenvorschlag für Otto-WagnerAreal. https://www.ots.at/presseaussendung/OTS_20130403_OTSo200/strache-kritisiert-expertenvorschlaege-fuer-otto-wagner-areal (letzter Zugriff am 5.12.2018).

APA-OTS FPÖ-Wien (14.2.2017): FP-Mahdalik wird rot-grünes Valentinstag-Massaker am Steinhof zur Anzeige bringen. https://www.ots.at/presseaussendung/OTS_20170214_ OTSo117/fp-mahdalik-wird-rot-gruenes-valentinstag-massaker-am-steinhof-zuranzeige-bringen (letzter Zugriff am 5.12.2018).

APA-OTS FPÖ-Wien (11.4.2017): FP-Mahdalik/Loibl: Baummord zahlt sich aus - Zerstörung von Steinhof beginnt. https://www.ots.at/presseaussendung/OTS_20170411_OTSoo83/ fp-mahdalikloibl-baummord-zahlt-sich-aus-zerstoerung-von-steinhof-beginnt (letzter Zugriff am 5.12.2018).

APA-OTS FPÖ Wien (19.4.2017): FP-Mahdalik zu Steinhof: Was zählt das Wort einer Vizebürgermeisterin? Genau nix! https://www.ots.at/presseaussendung/OTS_20170419_ OTSo113/fp-mahdalik-zu-steinhof-was-zaehlt-das-wort-einer-vizebuergermeisteringenau-nix (letzter Zugriff am 5.12.2018).

APA-OTS Initiative Denkmalschutz (2017): Initiative Denkmalschutz: Café-Restaurant „Schloss“ Cobenzl muss erhalten bleiben. Originalsubstanz aus 1910 weitgehend unversehrt. https://www.ots.at/presseaussendung/OTS_20170314_OTSoo05/initiativedenkmalschutz-caf-restaurant-schloss-cobenzl-muss-erhalten-bleiben-originalsubstanzaus-1910-weitgehend-unversehrt (letzter Zugriff am 5.12.2018).

Bonvalot, Michael (2018): Grazer AktivistInnen fordern Rettung von Augarten und Mur. http://www.bonvalot.net/grazer-aktivistinnen-fordern-rettung-von-augarten-undmur-832/ (letzter Zugriff am 5.12.2018).

DÖW Dokumentationsarchiv des österreichischen Widerstandes (2002): Das „Dritte Lager“ feiert Sonnenwende. http://www.doew.at/erkennen/rechtsextremismus/neues-vonganz-rechts/archiv/juni-2002/das-dritte-lager-feiert-sonnenwende (letzter Zugriff am 5.12.2018).

DÖW Dokumentationsarchiv des österreichischen Widerstandes (2004): „Sonnwendfeiern“. http://www.doew.at/erkennen/rechtsextremismus/neues-von-ganz-rechts/ archiv/juli-2004/sonnwendfeiern (letzter Zugriff am 5.12.2018).

DÖW Dokumentationsarchiv des österreichischen Widerstandes (2007): ÖLM-/WKRSonnwendfeier am Cobenzl. http://www.doew.at/erkennen/rechtsextremismus/neuesvon-ganz-rechts/archiv/juni-2007/oelm-wkr-sonnwendfeier-am-cobenzl (letzter Zugriff am 5.12.2018).

DÖW Dokumentationsarchiv des österreichischen Widerstandes (2016): Rechtsextreme feiern „Sonnenwende“. http://www.doew.at/erkennen/rechtsextremismus/neues-vonganz-rechts/archiv/juni-2016/rechtsextreme-feiern-sonnenwende (letzter Zugriff am 5.12.2018). 
FPÖ Wien (2017): Demo „Retten wir das Schloss Cobenzl“. https://www.fpoe-wien.at/ news-detail/artikel/demo-retten-wir-schloss-cobenzl/ (letzter Zugriff am 5.12.2018).

FPÖ Wien-Penzing (2017): freiheitliche Mandatare protestieren gegen Umweltzerstörung und Profitgier. https://penzing.fpoe-wien.at/news-detail/artikel/freiheitlichemandatare-protestieren-gegen-umweltzerstoerung-und-profitgier/ (letzter Zugriff am 5.12.2018).

Gedenkstätte Steinhof, ein Projekt des Dokumentationsarchivs des österreichischen Widerstandes (2018): Wien Steinhof. http://gedenkstaettesteinhof.at/de/ausstellung/wiensteinhof (letzter Zugriff am 5.12.2018).

Huber, Alexander (2018): FPÖ beschloss „unsinnigen Denkmalschutz“ selbst. https:// kurier.at/sport/fpoe-beschloss-unsinnigen-denkmalschutz-selbst/308.398.152 (letzter Zugriff am 5.12.2018).

Initiative Kultur- und Naturerbe Wien (2015): SOS Kulturerbe und Naturerbe Wien. http:// www.kulturerbewien.at/presse/ (letzter Zugriff am 5.12.2018).

Jäger, Siegfried (1997): Bemerkungen zur Durchführung von Diskursanalysen. http:// www.diss-duisburg.de/Internetbibliothek/Artikel/Durchfuehrung_Diskursanalyse.htm (letzter Zugriff am 5.12.2018).

Kadi, Justin (2016): Politische Auseinander-setzungen um das Otto-Wagner-Areal (Steinhof). https://urbanizm.net/4890231/politische-auseinandersetzungen-um-dasotto-wagner-areal-steinhof/ (letzter Zugriff 5.12.2018).

Klenk, Florian (2015): Wie die Wiener FPÖ einem NS-Opfer ins Ehrengrab spuckt. https:// www.falter.at/archiv/FALTER_20150729382E4FE539/wie-die-wiener-fpo-einem-nsopfer-ins-ehrengrab-spuckt (letzter Zugriff am 5.12.2018).

Krutzler, David (2017a): Otto-Wagner-Spital am Steinhof: Nachnutzung noch völlig unklar. https://derstandard.at/2000053887771/Otto-Wagner-Spital-am-SteinhofNachnutzung-noch-voellig-unklar (letzter Zugriff am 5.12.2018).

Krutzler, David (2017b): Wien: Café am Cobenzl zwangsgeräumt. https://derstandard. at/2000054136802/Stadt-Wien-startete-Zwangsraeumung-im-Restaurant-im-SchlossCobenzl (letzter Zugriff am 5.12.2018).

Laubner, Alexandra (2016): Der Kampf des Schlossherrn. https://www.wienerzeitung. at/nachrichten/wien/stadtpolitik/798023_Der-Kampf-des-Schlossherrn.html (letzter Zugriff am 5.12.2018).

Magistratsabteilungen 8 und 9 der Stadt Wien (2018a): Am Steinhof. https://www.geschichtewiki.wien.gv.at/Am_Steinhof (letzter Zugriff am 6.4.2019).

Magistratsabteilungen 8 und 9 der Stadt Wien (2018b): Cobenzl. https://www.geschichtewiki.wien.gv.at/Cobenzl (letzter Zugriff am 6.4.2019.

Magistratsabteilung 49 der Stadt Wien (2017): Erholungsgebiet Steinhofgründe. https:// www.wien.gv.at/umwelt/wald/erholung/steinhof.html (letzter Zugriff am 5.12.2018).

Magistratsabteilung 62 der Stadt Wien: Wien Gesamt Gemeinderatswahl 2015. https:// www.wien.gv.at/wahl/NET/GR151/GR151-109.htm (letzter Zugriff am 5.12.2018).

Magistratsdirektion der Stadt Wien (2018): Lebensqualität - Wien ist und bleibt Nummer 1. https://www.wien.gv.at/politik/international/vergleich/mercerstudie.html (letzter Zugriff 5.12.2018).

Schuh, Karin (2016): „Heritage Alert“: Hilfe für Steinhof-Initiative. https://diepresse.com/ home/panorama/wien/4907176/Heritage-Alert_Hilfe-fuer-SteinhofInitiative (letzter Zugriff am 5.12.2018).

Wiener Zeitung (2011): Steinhof Bebauung zum Teil abgesagt. https://www.wienerzeitung. at/nachrichten/wien/stadtpolitik/407807_Steinhof-Bebauung-zum-Teil-abgesagt.html (letzter Zugriff am 5.12.2018). 
